\title{
Risk Factors Associated with Mortality Among Patients with Novel Coronavirus Disease (COVID-19) in Africa
}

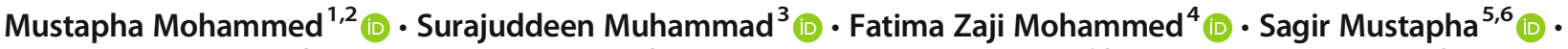 \\ Abubakar Sha'aban $^{1,2}$ (D) Najib Yahaya Sani ${ }^{7,8}$ (D) Mubarak Hussaini Ahmad ${ }^{6,9}$ (D) Auwal Adam Bala $^{10,11}$ (D) \\ Marzuq Abubakar Ungogo ${ }^{12,13}$ (D) Nawaf M Alotaibi $^{14}$ (D) Hadzliana Zainal $^{1}$ (D)
}

Received: 1 September 2020 / Revised: 22 September 2020 / Accepted: 28 September 2020 / Published online: 13 October 2020

(C) W. Montague Cobb-NMA Health Institute 2020

\begin{abstract}
Background The novel coronavirus disease (COVID-19) caused by severe acute respiratory syndrome coronavirus 2 (SARS-CoV2) was first reported in China and later spread rapidly to other parts of the world, including Africa. Africa was projected to be devastated by COVID-19. There is currently limited data regarding regional predictors of mortality among patients with COVID-19. This study aimed to evaluate the independent risk factors associated with mortality among patients with COVID-19 in Africa.

Methods A total of 1028 confirmed cases of COVID-19 from Africa with definite survival outcomes were identified retrospectively from an open-access individual-level worldwide COVID-19 database. The live version of the dataset is available at https:// github.com/beoutbreakprepared/nCoV2019. Multivariable logistic regression was conducted to determine the risk factors that independently predict mortality among patients with COVID-19 in Africa.

Results Of the 1028 cases included in study, 432 (42.0\%) were females with a median (interquartile range, IQR) age of 50 (24) years. Older age (adjusted odds ratio $\{\mathrm{aOR}\}$ 1.06; [95\% confidence intervals $\{95 \% \mathrm{CI}\}, 1.04-1.08]$ ), presence of chronic disease (aOR 9.63; [95\% CI, 3.84-24.15]), travel history (aOR 2.44; [95\% CI, 1.26-4.72]), as well as locations of Central Africa (aOR 0.14; [95\% CI, 0.03-0.72]) and West Africa (aOR 0.12; [95\% CI, 0.04-0.32]) were identified as the independent risk factors significantly associated with increased mortality among the patients with COVID-19.

Conclusions The COVID-19 pandemic is evolving gradually in Africa. Among patients with COVID-19 in Africa, older age, presence of chronic disease, travel history, and the locations of Central Africa and West Africa were associated with increased mortality. A regional response should prioritize strategies that will protect these populations. Also, conducting a further in-depth study could provide more insights into additional factors predictive of mortality in COVID-19 patients.
\end{abstract}

Keywords Africa $\cdot$ COVID-19 $\cdot$ Mortality $\cdot$ Pandemic $\cdot$ Risk factors $\cdot$ SARS-COV-2

Mustapha Mohammed

macreener88@gmail.com

1 School of Pharmaceutical Sciences, Universiti Sains Malaysia, Penang, Pulau Pinang, Malaysia

2 Department of Clinical Pharmacy and Pharmacy Practice, Ahmadu Bello University, Zaria, Kaduna, Nigeria

3 Faculty of Veterinary Medicine, Ahmadu Bello University, Zaria, Kaduna, Nigeria

4 School of Dental Health Sciences, Shehu Idris College of Health Sciences and Technology, Makarfi, Kaduna, Nigeria

5 School of Medical Sciences, Universiti Sains Malaysia, Kubang Kerian, Kelantan, Malaysia

6 Department of Pharmacology and Therapeutics, Ahmadu Bello University, Zaria, Kaduna, Nigeria

7 Center for Drug Research, Universiti Sains Malaysia, Penang, Pulau Pinang, Malaysia
8 Department of Pharmaceutical and Medicinal Chemistry, Bayero University Kano, Kano, Nigeria

9 School of Pharmacy Technician, Aminu Dabo College of Health Sciences and Technology, Kano, Nigeria

10 Department of Pharmacology, College of Medicine and Health Sciences, Federal University Dutse, Jigawa, Nigeria

11 Department of Pharmacology and Therapeutics, Bayero University Kano, Kano, Nigeria

12 Department of Veterinary Pharmacology and Toxicology, Ahmadu Bello University, Zaria, Nigeria

13 Institute of Infection, Immunity and Inflammation, College of Medical, Veterinary and Life Sciences, University of Glasgow, Glasgow, UK

14 College of Pharmacy, Northern Border University, Rafha, Kingdom of Saudi Arabia 


\section{Introduction}

Coronavirus disease 2019 (COVID-19) is an infectious disease caused by severe acute respiratory syndrome coronavirus 2 (SARS-CoV-2) [1]. The first case of the virus was reported in Wuhan, China, in December 2019 [2, 3]. The virus has spread rapidly worldwide since its outbreak with the World Health Organization (WHO) declaring it as a pandemic [4]. As of August 25, 2020, more than 23 million cases of COVID-19 have been reported across over 215 countries/territories, resulting in more than 800 thousand deaths [5]. The global COVID-19 data showed America, Asia, and Europe to be the most hit continents with COVID-19 as compared with other continents like Africa [6]. The first confirmed case of COVID-19 in Africa was reported in Egypt on February 14, 2020 [7]. The African countries currently identified to have the highest number of cases are South Africa, Egypt, Morocco, Nigeria, and Ghana [5].

Africa is the largest, most populous continent globally, after Asia, with the youngest and most diverse population with considerable genetic, linguistic, cultural, and ethnic diversity [8-11]. Africa is also the least-developed continent, contributing about $40 \%$ of the global burden of disease [12]. The major contributors to the African disease burden include infectious diseases (HIV and AIDS, malaria), neglected tropical diseases (trypanosomiasis, leishmaniasis), diseases predominantly affecting African populations (sickle cell disease), non-communicable diseases (hypertension, diabetes), and diseases of poverty (malnutrition) [12]. These disease burdens are further complicated by limited access to safe, effective, quality, and affordable medicines. Responses to African epidemics have been threatened by insufficient infrastructure and weak healthcare systems, including lack of sufficient monitoring to determine the magnitude of the outbreak and insufficient structures to prevent, diagnose, and treat diseases [13]. There is a high reliance on traditional, complementary, and alternative medicines in Africa [11, 14]. Deficient healthcare systems in Africa have resulted in high preventable morbidity and mortality, creating a vicious cycle of poverty [15-17]. These factors, put together, led to the projections that the COVID-19 pandemic could be challenging to keep under control in Africa and could cause a substantial socioeconomic burden if allowed to spread [18]. Therefore, in this study, we described the characteristics of patients with COVID-19 in Africa and identified the various risk factors associated with COVID-19 mortality in the continent.

\section{Methods}

\section{Study Design}

The study was a retrospective cross-sectional study that involved the extraction of all confirmed cases of COVID-19 in
Africa that had a definite survival outcome from the openaccess individual-level COVID-19 database reported elsewhere [19]. The data were extracted on August 25, 2020. The database is a hub of updated and curated COVID-19 data generated from a range of different validated sources, including international, national, state, provincial, municipal, news reports, as well as peer-reviewed articles. A real-time updated version of the dataset is downloadable as a commaseparated value (CSV) from a GitHub repository (https:// github.com/beoutbreakprepared/nCoV2019). The repository is a product of the Open COVID-19 Data Working Group: a multi-organizational global scheme that focused on enabling rapid sharing of trusted and openaccess public health data in advancing the response to infectious diseases.

\section{Data Collection}

The patient characteristics collected in our study include COVID-19 diagnosis confirmation, demographic data such as age and gender, chronic disease, travel history, and survival outcomes (dead or alive). Survival (alive) was defined as those patients with outcomes labeled as "alive," "discharge," "discharged," "discharged from hospital," "recovered," "recovering from home," "released from quarantine," "stable," or "stable condition," while mortality (dead) was defined as those patients with outcomes labeled as "dead," "died," "death," or "deceased."

\section{Inclusion and Exclusion Criteria}

We included only reported confirmed cases of COVID-19 that had a definite survival outcome (dead or alive) and from only African countries. Those confirmed cases without survival outcomes and not from any of the African countries were excluded from this study.

\section{Statistical Analysis}

The data collected in CSV format were exported into Microsoft Excel and then analyzed with IBM SPSS Version 24.0 (SPSS Inc., Chicago, IL, USA). The data were assessed for missing values, and a multiple imputation method was used to handle variables with missing data of more than 5\%. Five imputations were performed and combined through Rubin's rules. A normality test was conducted on the continuous numerical variable, age, using the histogram method and Kolmogorov-Smirnov (KS) test, and was found to be not normally distributed. The results of age were presented as median (interquartile range, IQR), while the categorical variables were presented as frequencies and percentages. 
The risk factors associated with mortality were determined using multivariable logistic regression (MLR). Univariable analysis was initially conducted using simple binary logistic regression (SLR) to screen each of the independent variables (age, gender, chronic disease, travel history, region) for inclusion in the multivariable analysis. From the results of SLR, variables that had a $p<0.25$ and those considered relevant were included in the MLR. The MLR was performed using both the backward and forward likelihood ratio method to determine independent risk factors associated with mortality. Multicollinearity and interactions between the variables were checked. Goodness-of-fit model assumptions were checked using Hosmer-Lemeshow and Omnibus Tests of Model Coefficients. The results of the SLR were presented as a crude odds ratio (OR) with $95 \%$ confidence intervals (CIs) and corresponding $p$ values, while the final model was presented as adjusted OR with $95 \%$ CIs and corresponding $p$ values.

\section{Results}

\section{Characteristics of Patients with COVID-19}

A total of 1028 COVID-19 patients from Africa were found to have a definitive survival outcome. The number of female patients were $432(42.0 \%)$, with an overall median (IQR) age of 50 (24) years. Among the patients, $32(3.1 \%)$ had at least one chronic disease, and 210 (20.4\%) had travel history. Most of the COVID-19 patients were from West Africa, 479 (46.6\%), and the least were from Central Africa 89 (8.7\%). From the available data, the overall number of deaths was 60 (5.6\%). The details of the characteristics of patients with COVID-19 are presented in Table 1 .

\begin{tabular}{llc} 
Table 1 Demographic & & \\
\cline { 2 - 3 } $\begin{array}{l}\text { and clinical } \\
\text { characteristics of the } \\
\text { patients with COVID-19 }\end{array}$ & Characteristics & $N(\%)$ \\
\cline { 2 - 3 } & Age (median, IQR) & $50(24)$ \\
& Female gender & $432(42.0)$ \\
& Chronic disease & $32(3.1)$ \\
& Travel history & $210(20.4)$ \\
& African region & \\
& North & $90(8.8)$ \\
& South & $196(19.1)$ \\
& Central & $89(8.7)$ \\
& East & $174(16.9)$ \\
& West & $479(46.6)$ \\
& Mortality & $60(5.6)$ \\
\hline
\end{tabular}

\section{Risk Factors Associated of Mortality in Patients with COVID-19}

From the univariable analysis, all the five independent variables (age, gender, chronic disease, travel history, and region) were selected for the multivariable analysis. The final model of variables associated with mortality in patients with COVID-19 showed that the predictors of the mortality were older age (aOR 1.06; [95\% CI, 1.04-1.08]), presence of chronic disease (aOR 9.63; [95\% CI, 3.84-24.15]), and travel history (aOR 2.44; [95\% CI, 1.26-4.72]). Also, patients with COVID-19 located in Central Africa (aOR 0.14; [95\% CI, 0.03-0.72]) and West Africa (aOR 0.12; [95\% CI, 0.04$0.32]$ ) were also associated with mortality. No possible collinearity in the included variables was observed and no interactions between the variables. The model fit for the data was excellent, as the Hosmer-Lemeshow test $p$ value was 0.55 . The univariate analysis and the final model were presented as ORs or adjusted ORs with 95\% CIs and corresponding $p$ values in Table 2 .

\section{Discussion}

We provide in this study novel findings on the risk factors that are independently associated with mortality among 1028 patients with COVID-19 in Africa. Africa has been projected to be devastated by COVID-19 [20, 21]. It was suggested that with neither effective treatment nor vaccines, and without prior immunity, the impact of the virus might be devastating because of the health systems challenges in the continent [20]. However, the current evidence suggests that Africa is one of the least hits by COVID-19, accounting for only about $5 \%$ and $3 \%$ of the world's cumulative cases and deaths, respectively [5]. A weak healthcare system, low socioeconomic status, healthcare-seeking, cultural and cohabitation practices, variations in immune profiles, and limited technology could be attributed to the limited impact of COVID-19 in Africa [22].

In this study, we identified the risk factors that are independently associated with mortality in patients with COVID-19 in Africa from a worldwide open-access database reported elsewhere [19]. The COVID-19 mortality rate reported previously ranged from 4 to $28 \%$ [23-28]. The lower mortality rate of $5.6 \%$ in our study is consistent with the previous studies and could be explained by the milder burden of COVID-19 in Africa. This variation in mortality rates may be due to discrepancies in healthcare systems, public health responses, and/or epidemiological characteristics of patients [29]. Other reports suggested lower contact tracing, weaker testing capacities, and poor reporting strategies in Africa could be responsible for the lower reported cases of COVID-19 [21, 30]. 
Table 2 Risk factors associated with mortality in patients with COVID-19

\begin{tabular}{|c|c|c|c|c|}
\hline \multirow[t]{2}{*}{ Variable } & \multicolumn{2}{|l|}{ Univariate analysis } & \multicolumn{2}{|c|}{ Multivariable analysis } \\
\hline & OR $(95 \% \mathrm{CI})$ & $p$ value & aOR $(95 \% \mathrm{CI})$ & $p$ value \\
\hline Age, years & $1.06(1.04-1.08)$ & $<0.001$ & $1.06(1.04-1.08)$ & $<0.001$ \\
\hline Female gender & $0.99(0.58-1.67)$ & 0.954 & - & - \\
\hline Chronic disease & $16.06(7.52-34.30)$ & $<0.001$ & $9.63(3.84-24.15)$ & $<0.001$ \\
\hline Travel history & $3.78(2.22-6.43)$ & $<0.001$ & $2.44(1.26-4.72)$ & 0.008 \\
\hline \multicolumn{5}{|l|}{ African region } \\
\hline North & 1 & - & 1 & - \\
\hline South & $0.15(0.22-1.26)$ & 0.147 & $0.38(0.14-1.06)$ & 0.066 \\
\hline Central & $0.18(0.04-0.87)$ & 0.032 & $0.14(0.03-0.72)$ & 0.018 \\
\hline East & $1.34(0.61-2.93)$ & 0.461 & $0.44(0.16-1.18)$ & 0.103 \\
\hline West & $0.19(0.08-0.46)$ & $<0.001$ & $0.12(0.04-0.32)$ & $<0.001$ \\
\hline
\end{tabular}

OR odds ratio, $a O R$ adjusted odds ratio, $95 \%$ CI 95\% confidence interval; classification table $94.2 \%$ correctly classified using backward stepwise (likelihood ratio) method of the logistic regression
We identified several risk factors associated with mortality in patients with COVID-19 in Africa, including older age, chronic disease, travel history, and patients in Central and West Africa. Similar studies reported a significant increase in mortality among older COVID-19 patients in Wuhan, China [27], New York City, the USA [31], Ontario, Canada [32], Lombardy, Italy [33], and worldwide [28]. Older age has also been reported as a key independent predictor of mortality in similar diseases like SARS and MERS [34, 35]. A higher viral load was found in older patients with COVID-19 [36] and could be associated with mortality. Previous studies also found that older subjects had improved innate host responses to inoculated SARS-CoV virus than younger ones, with an increased expression of genes associated with inflammation and reduced expression of type I interferon-beta [37]. Also, the age-dependent defects in the function of cellular immunity and the overproduction of type 2 cytokines could lead to reduced control of viral replication and more prolonged proinflammatory responses, leading to poor outcomes, including death [38]. Thus, older age is associated with a weaker immune system and are more susceptible to the infections, acute respiratory distress syndrome (ARDS), and, subsequently, death.

The studies on patients with COVID-19 infection have shown that those with underlying chronic diseases not only have a higher risk of developing the disease but also are more likely to die from the infection [39]. Chronic disease conditions, such as cardiovascular and metabolic diseases, were reported to be associated with severe cases of COVID-19 [40, 41]. Chronic obstructive pulmonary disease, hypercholesterolemia, obesity, and diabetes were reported to be independently associated with mortality in COVID-19 patients $[26,33]$. Similarly, our study identified that the presence of underlying chronic disease conditions is independently associated with mortality in patients with COVID-19. Conversely, a study found no association between in-hospital mortality and some common coexisting medical conditions, including hypertension, diabetes, or cancer [31].
Our study reports travel history to be associated with mortality in patients with COVID-19. Several person-to-person transmission of SARS-CoV-2 have been documented in patients with travel-related COVID-19 [42]. Initially, the virus was first imported to Africa, like many other continents, with most imported cases arriving from Europe and the USA rather than from China [43]. Several preventive measures have been implemented in different countries of Africa, including restrictions on travel, flight cancelations, restrictions on mass gathering, school closures, and border closures [43]. There are ongoing concerns over the effectiveness of travel restrictions to contain the spread of COVID-19. A study found that travel restrictions had only moderately improved the initial spread of COVID-19 and could only considerably reduce transmissions if combined with other prevention and control measures [44].

This study has some limitations and should be interpreted with caution. The study was retrospective, and data utilized was from an open-access curated and validated COVID-19 data repository. Thus, the data is secondary, and other clinical and laboratory data to assess patient disease status were unavailable. Smoking status and other socioeconomic status were also lacking. The severity of the chronic conditions and compliance with medications were not documented and could not be evaluated. Furthermore, the cases and mortality may not reflect the actual percentage because only patients with definitive outcomes were included resulting in relatively smaller numbers than the real confirmed cases reported in Africa.

\section{Conclusion}

The COVID-19 pandemic continues to evolve gradually in Africa despite higher projections. We found that older age, chronic disease, travel history, and location of Central and West Africa were risk factors associated with death in patients with COVID-19 in Africa. A regional response should 
prioritize strategies that will protect these categories of patients. Also, conducting a further in-depth study could provide more insights into additional factors predictive of mortality in COVID-19 patients. Government intervention should ensure improved surveillance, laboratory capacity, and public health response that could further validate the findings of COVID-19 studies in Africa.

\section{Compliance with Ethical Standards}

Conflict of Interest The authors declare that they have no conflict of interest.

Ethics Approval All procedures performed in studies involving human participants were in accordance with the ethical standards of the institutional research committee and with the 1964 Helsinki declaration and its later amendments or comparable ethical standards.

Informed Consent The study utilized secondary data as such informed consent could not be obtained.

\section{References}

1. Lai C-C, Shih T-P, Ko W-C, Tang H-J, Hsueh P-R. Severe acute respiratory syndrome coronavirus 2 (SARS-CoV-2) and corona virus disease-2019 (COVID-19): the epidemic and the challenges. Int J Antimicrob Agents. 2020;105924.

2. Zhu N, Zhang D, Wang W, Li X, Yang B, Song J, et al. A novel coronavirus from patients with pneumonia in China, 2019. N Engl J Med. 2020;382(8):727-33. https://doi.org/10.1056/ NEJMoa2001017.

3. Surveillances $\mathrm{V}$. The epidemiological characteristics of an outbreak of 2019 novel coronavirus diseases (COVID-19) - China, 2020. China CDC Wkly. 2020;2(8):113-22.

4. Cucinotta D, Vanelli M. WHO declares COVID-19 a pandemic. Acta Bio-Med: Atenei Parmensis. 2020;91(1):157-60.

5. Dong E, Du H, Gardner L. An interactive web-based dashboard to track COVID-19 in real time. Lancet Infect Dis. 2020;20(5):533-4. https://doi.org/10.1016/S1473-3099(20)30120-1.

6. Spiteri G, Fielding J, Diercke M, Campese C, Enouf V, Gaymard A, et al. First cases of coronavirus disease 2019 (COVID-19) in the WHO European region, January 24 to February 212020. Eurosurveillance. 2020;25(9):2000178.

7. Medhat MA, El Kassas M. COVID-19 in Egypt: uncovered figures or a different situation? J Glob Health. 2020;10(1):010368. https:// doi.org/10.7189/jogh.10.010368.

8. Cooper F. Africa since 1940: the past of the present: Cambridge University Press; 2019.

9. Nzamujo F. A new approach to sustainable livelihoods: African youth and agriculture. Development. 1999;42(2):64-7.

10. Iliffe J. Africans: the history of a continent: Cambridge University Press; 2017.

11. Mohammed M, Umar FI, Labaran KS, Sha'aban A, Abdu-Aguye $\mathrm{SN}, \mathrm{Ma}$ 'aji $\mathrm{HU}$, et al. Evaluation of adverse drug reaction reporting among traditional medicine practitioners in Zaria Kaduna State Nigeria. Trop J Nat Prod Res. 2019;3(3):85-90. https://doi.org/10. 26538/tjnpr/v3i3.4.

12. Lopez AD, Mathers CD, Ezzati M, Jamison DT, Murray CJ. Global and regional burden of disease and risk factors, 2001: systematic analysis of population health data. Lancet. 2006;367(9524):174757.
13. Rosenthal PJ, Breman JG, Djimde AA, John CC, Kamya MR, Leke RGF, et al. COVID-19: shining the light on Africa. Am J Trop Med Hyg. 2020;102(6):1145-8. https://doi.org/10.4269/ajtmh.20-0380.

14. Hill J, Mills C, Li Q, Smith JS. Prevalence of traditional, complementary, and alternative medicine use by cancer patients in low income and lower-middle income countries. Global Public Health. 2019;14(3):418-30.

15. Oluwatayo IB, Ojo AO. Walking through a tightrope: the challenge of economic growth and poverty in Africa. J Dev Areas. 2018;52(1):59-69.

16. Adeyeye SAO, Adebayo-Oyetoro AO, Tiamiyu HK. Poverty and malnutrition in Africa: a conceptual analysis. Nutrition \& Food Science. 2017;47:754-64.

17. White H, Killick T, Savane M-A, Kayizzi-Mugerwa S. African poverty at the millennium: causes, complexities, and challenges. The World Bank; 2001.

18. Zhao Z, Li X, Liu F, Zhu G, Ma C, Wang L. Prediction of the COVID-19 spread in African countries and implications for prevention and control: a case study in South Africa, Egypt, Algeria, Nigeria, Senegal and Kenya. Sci Total Environ. 2020;729: 138959. https://doi.org/10.1016/j.scitotenv.2020.138959.

19. Xu B, Gutierrez B, Mekaru S, Sewalk K, Goodwin L, Loskill A, et al. Epidemiological data from the COVID-19 outbreak, real-time case information. Sci Data. 2020;7(1):106. https://doi.org/10.1038/ s41597-020-0448-0.

20. Nkengasong JN, Mankoula W. Looming threat of COVID-19 infection in Africa: act collectively, and fast. Lancet. 2020;395(10227):841-2.

21. Zhao Z, Li X, Liu F, Zhu G, Ma C, Wang L. Prediction of the COVID-19 spread in African countries and implications for prevention and controls: a case study in South Africa, Egypt, Algeria, Nigeria, Senegal and Kenya. Sci Total Environ. 2020;138959.

22. Pareek M, Bangash MN, Pareek N, Pan D, Sze S, Minhas JS, et al. Ethnicity and COVID-19: an urgent public health research priority. Lancet. 2020;395(10234):1421-2.

23. Huang C, Wang Y, Li X, Ren L, Zhao J, Hu Y, et al. Clinical features of patients infected with 2019 novel coronavirus in Wuhan, China. Lancet. 2020;395(10223):497-506. https://doi. org/10.1016/S0140-6736(20)30183-5.

24. Chen N, Zhou M, Dong X, Qu J, Gong F, Han Y, et al. Epidemiological and clinical characteristics of 99 cases of 2019 novel coronavirus pneumonia in Wuhan, China: a descriptive study. Lancet. 2020;395(10223):507-13. https://doi.org/10.1016/ S0140-6736(20)30211-7.

25. Chen T, Wu D, Chen H, Yan W, Yang D, Chen G, et al. Clinical characteristics of 113 deceased patients with coronavirus disease 2019: retrospective study. BMJ. 2020;368:m1091. https://doi.org/ 10.1136/bmj.m1091.

26. Wang D, Hu B, Hu C, Zhu F, Liu X, Zhang J, et al. Clinical characteristics of 138 hospitalized patients with 2019 novel coronavirus-infected pneumonia in Wuhan, China. Jama. 2020;323(11):1061-9. https://doi.org/10.1001/jama.2020.1585.

27. Zhou F, Yu T, Du R, Fan G, Liu Y, Liu Z, et al. Clinical course and risk factors for mortality of adult inpatients with COVID-19 in Wuhan, China: a retrospective cohort study. Lancet. 2020;395(10229):1054-62. https://doi.org/10.1016/S01406736(20)30566-3.

28. Cooperberg PL, Robertson WD, Fry P, Sweeney V. High resolution real time ultrasound of the carotid bifurcation. J Clin Ultrasound. 1979;7(1):13-7. https://doi.org/10.1002/jcu.1870070107.

29. Kim D-H, Choe YJ, Jeong J-Y. Understanding and interpretation of case fatality rate of coronavirus disease 2019. J Korean Med Sci. 2020;35(12).

30. Hopman J, Allegranzi B, Mehtar S. Managing COVID-19 in lowand middle-income countries. Jama. 2020;323(16):1549-50. https://doi.org/10.1001/jama.2020.4169. 
31. Mikami T, Miyashita H, Yamada T, Harrington M, Steinberg D, Dunn A, et al. Risk factors for mortality in patients with COVID-19 in New York City. J Gen Intern Med. 2020. https://doi.org/10.1007/ s11606-020-05983-z.

32. Fisman DN, Bogoch I, Lapointe-Shaw L, McCready J, Tuite AR. Risk factors associated with mortality among residents with coronavirus disease 2019 (COVID-19) in long-term care facilities in Ontario, Canada. JAMA Netw Open. 2020;3(7):e2015957. https:// doi.org/10.1001/jamanetworkopen.2020.15957.

33. Grasselli G, Greco M, Zanella A, Albano G, Antonelli M, Bellani $\mathrm{G}$, et al. Risk factors associated with mortality among patients with COVID-19 in intensive care units in Lombardy, Italy. JAMA Intern Med. 2020. https://doi.org/10.1001/jamainternmed.2020.3539.

34. Choi KW, Chau TN, Tsang O, Tso E, Chiu MC, Tong WL, et al. Outcomes and prognostic factors in 267 patients with severe acute respiratory syndrome in Hong Kong. Ann Intern Med. 2003;139(9):715-23. https://doi.org/10.7326/0003-4819-139-9200311040-00005.

35. Hong KH, Choi JP, Hong SH, Lee J, Kwon JS, Kim SM, et al. Predictors of mortality in Middle East respiratory syndrome (MERS). Thorax. 2018;73(3):286-9. https://doi.org/10.1136/ thoraxjnl-2016-209313.

36. To KK, Tsang OT, Leung WS, Tam AR, Wu TC, Lung DC, et al. Temporal profiles of viral load in posterior oropharyngeal saliva samples and serum antibody responses during infection by SARSCoV-2: an observational cohort study. Lancet Infect Dis. 2020;20(5):565-74. https://doi.org/10.1016/S1473-3099(20) 30196-1.

37. Smits SL, de Lang A, van den Brand JM, Leijten LM, IJcken v IWF, Eijkemans MJ, et al. Exacerbated innate host response to SARS-CoV in aged non-human primates. PLoS Pathog. 2010;6(2):e1000756. https://doi.org/10.1371/journal.ppat. 1000756
38. Opal SM, Girard TD, Ely EW. The immunopathogenesis of sepsis in elderly patients. Clin Infect Dis. 2005;41(Supplement_7):S504 12. https://doi.org/10.1086/432007.

39. Verity R, Okell LC, Dorigatti I, Winskill P, Whittaker C, Imai N, et al. Estimates of the severity of coronavirus disease 2019: a model-based analysis. Lancet Infect Dis. 2020;20(6):669-77. https://doi.org/10.1016/S1473-3099(20)30243-7.

40. Zhang JJ, Dong X, Cao YY, Yuan YD, Yang YB, Yan YQ, et al. Clinical characteristics of 140 patients infected with SARS-CoV-2 in Wuhan, China. Allergy. 2020;75(7):1730-41. https://doi.org/10. 1111/all.14238.

41. Li B, Yang J, Zhao F, Zhi L, Wang X, Liu L, et al. Prevalence and impact of cardiovascular metabolic diseases on COVID-19 in China. Clin Res Cardiol: Off J German Cardiac Soc. 2020;109(5): 531-8. https://doi.org/10.1007/s00392-020-01626-9.

42. Chu DK, Akl EA, Duda S, Solo K, Yaacoub S, Schunemann HJ, et al. Physical distancing, face masks, and eye protection to prevent person-to-person transmission of SARS-CoV-2 and COVID-19: a systematic review and meta-analysis. Lancet. 2020;395(10242): 1973-87. https://doi.org/10.1016/S0140-6736(20)31142-9.

43. Gilbert M, Pullano G, Pinotti F, Valdano E, Poletto C, Boelle PY, et al. Preparedness and vulnerability of African countries against importations of COVID-19: a modelling study. Lancet. 2020;395(10227):871-7. https://doi.org/10.1016/S0140-6736(20) 30411-6.

44. Chinazzi M, Davis JT, Ajelli M, Gioannini C, Litvinova M, Merler S, et al. The effect of travel restrictions on the spread of the 2019 novel coronavirus (COVID-19) outbreak. Science. 2020;368(6489):395-400. https://doi.org/10.1126/science. aba9757.

Publisher's Note Springer Nature remains neutral with regard to jurisdictional claims in published maps and institutional affiliations. 